\title{
Un enfoque de control de movimiento de un helicóptero con cuatro rotores
}

\author{
H. Yáñez-Badillo ${ }^{1}$, R. Tapia-Olvera ${ }^{2}$, F. Beltran-Carbajal ${ }^{3}$, O. Aguilar-Mejía ${ }^{1}$, \\ I. López-García ${ }^{3}$ \\ ${ }^{1}$ Universidad Politécnica de Tulancingo, \\ Departamento de Posgrado, Tulancingo, Hidalgo, \\ México \\ ${ }^{2}$ Universidad Nacional Autónoma de México, \\ Departamento de Ingeniería Eléctrica, Ciudad de México, \\ México \\ ${ }^{3}$ Universidad Autónoma Metropolitana, \\ Unidad Azcapotzalco, Departamento de Energía, Ciudad de México, \\ México \\ \{dopthugo.yanez,omar.aguilar\}@upt.edu.mx, rtapia@fi-b.unam.mx, \\ \{fbeltran,ilg\}@azc.uam.mx
}

\begin{abstract}
Resumen. En este artículo se describe un esquema de control para tareas de regulación y seguimiento de trayectorias planificadas para el movimiento de un helicóptero con cuatro rotores, el cual también se conoce como Quadrotor. El esquema de control consiste de cuatro controladores clásicos y una etapa de generación de trayectorias para el movimiento de traslación deseado en el plano $X-Y$. Se incluyen resultados de simulación donde se demuestra que el desempeño del esquema de control de movimiento especificado para el Quadrotor es aceptable.
\end{abstract}

Palabras clave: Quadrotor, control de movimiento, seguimiento de trayectorias, sistemas mimo.

\section{A Motion Control Approach of a Helicopter with Four Rotors}

\begin{abstract}
In this paper a control scheme is described for regulation and trajectory tracking tasks planned for the motion of a helicopter with four rotors wich is also known as Quadrotor. The control scheme consists of four classical controllers and a generation stage of trajectories for the desired translational movement in the $X-Y$ plane. Simulations results show the acceptable performance of the control scheme specified for the Quadrotor.
\end{abstract}

Keywords: Quadrotor, motion control, trajectory tracking, mimo systems. 


\section{Introducción}

El interés en el estudio de los vehículos aéreos no tripulados (UAV's, por sus siglas en inglés) se ha incrementado en los últimos años. Una de las principales plataformas en la que se han enfocado diversas investigaciones es el helicóptero con cuatro rotores comúnmente llamado Quadrotor. Éste tiene la capacidad de aterrizar y despegar verticalmente (VTOL, por sus siglas en inglés) característica que permite su operación en interiores de manera segura, tarea complicada para otro tipo de UAV's como los de ala fija [1].

En la literatura se han realizado algunas consideraciones fundamentales para el análisis de esta plataforma. En la mayoría de las contribuciones el Quadrotor se considera como un cuerpo rígido, por lo que se deben emplear dos marcos de referencia para describir su comportamiento dinámico. El primer marco de referencia se establece fijo respecto a la superficie terrestre $O_{e}$ y el segundo solidario al cuerpo del robot $O_{b}$ [2]. Existen diversas representaciones matemáticas para describir la dinámica del robot, las citadas en la literatura son los formalismos: Euler-Lagrange [3] y Newton-Euler [4], esta última es la adoptada para el presente estudio.

Las técnicas para regular el seguimiento de trayectorias son diversas, entre las principales se tienen a los controladores convencionales PD, PI y PID [5-9], controladores no lineales del tipo backstepping [10,11], modos deslizantes [12,13], controladores robustos $H \infty$ [14], difusos [15], redes neuronales [16], algoritmos híbridos $[17,18]$ y control por planitud diferencial [19,20]. El presente artículo hace énfasis en los métodos de control clásicos, principalmente para remarcar sus versatilidad y facilidad de implementación, así como la funcionalidad para fundamentar métodos de control efectivos.

En [5] se presentan controladores PD y PID para la regulación del movimiento de rotación del robot, donde no se han considerado los acoples dinámicos provocados por los efectos giroscópicos del sistema. Los parámetros del controlador fueron sintonizados mediante simulaciones previas a la implementación, donde se concluye que las aproximaciones clásicas de control son suficientes para estabilizar el Quadrotor en condiciones de vuelo estacionario. Sin embargo, ante la presencia de perturbaciones de gran magnitud estos algoritmos no son capaces de estabilizar el sistema. En [6] se trabaja con controladores PI en cascada, donde la regulación de los principales movimientos del helicóptero se llevan a cabo a través del ajuste de las velocidades angulares de los cuatro rotores que constituyen el mecanismo. Por otro lado, en [7] se desarrolla un controlador PD mejorado, que se enfoca en la respuesta transitoria de un micro helicóptero de cuatro rotores ya que debido a sus características dinámicas es sensible incluso a pequeñas perturbaciones. En el trabajo se emplean los cuaternios para evitar singularidades en el modelo y el error de esta representación es minimizado con las ganancias del controlador, de tal forma que no afecten el desempeño del vuelo del Quadrotor. En [8] se establecen dos controladores PD para controlar los desplazamientos en las direcciones $X$ y $Y$, mediante los ángulos $\theta$ y $\phi$ respectivamente. Una propuesta de un controlador de altitud PID restringido mediante técnicas de regulación cuadráticas (LQR) se propone en 
[9]; el algoritmo propuesto se presenta de esta manera para que las ganancias del controlador PID sean determinadas dentro de las posibilidades físicas del sistema, sin degradar la acción del controlador mediante una función de costo.

A pesar de ser sensible a perturbaciones, los controladores clásicos presentan una estructura compacta y funcional con resultados satisfactorios, lo que ha permitido su implementación para modelos comerciales [18]. En la literatura es posible encontrar la estructura general de estos algoritmos aunado a un grado de robustez mediante técnicas como el análisis de backstepping [21], redes neuronales [22], por mencionar algunos, donde las ganancias del controlador se actualizan de forma dinámica, sin afectar la estabilidad del robot.

En este artículo se describe un esquema de control para tareas de regulación y seguimiento de trayectorias planificadas para el movimiento de un helicóptero con cuatro rotores. El esquema de control consiste de cuatro controladores clásicos y de una etapa de generación de trayectorias para el movimiento de traslación deseado en el plano $X-Y$. Se incluyen algunos resultados de simulación para mostrar el desempeño aceptable del esquema de control de movimiento especificado para el Quadrotor.

\section{Dinámica de un helicóptero con cuatro rotores}

Esta clase de vehículo aéreo presenta seis grados de libertad, mediante cuatro entradas de control principales que dependen de las velocidades angulares de cada rotor. El movimiento en el plano cartesiano se logra mediante movimientos de alabeo (roll), cabeceo ( pitch) y giñada (yaw) que relacionan los ángulos de Tait-Bryan $\phi, \theta$ y $\psi$ (utilizados en el área de aeronáutica debido a su configuración), con los desplazamientos en las direcciones $X, Y$ y $Z$, respectivamente. El sentido de la rotación de las aspas para cada rotor $\omega_{i}, i=1, \ldots, 4$, deben de establecerse mediante pares, es decir $\omega_{1}$ y $\omega_{3}$ en el sentido conforme a las manecillas del reloj y $\omega_{2}$ y $\omega_{4}$ en sentido contrario al de las manecillas del reloj; la rotaciones de las hélices en contacto con el aire, producen fuerzas y pares que le permiten el movimiento a esta clase de vehículos aéreos (véase la Fig. 1).
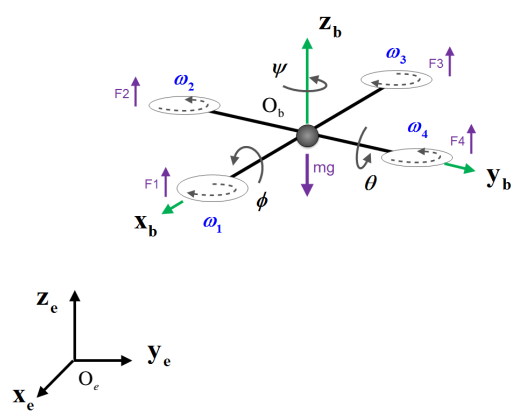

Fig. 1. Diagrama de cuerpo libre de un Quadrotor. 
El modelo matemático que describe la dinámica del helicóptero con cuatro rotores está dado por [4]

$$
\begin{aligned}
& \ddot{x}=\frac{1}{m}(\sin \theta \cos \phi \cos \psi+\sin \phi \sin \psi) u_{1}-\frac{c_{x}}{m} \dot{x}, \\
& \ddot{y}=\frac{1}{m}(\sin \theta \sin \psi \cos \phi-\sin \phi \cos \psi) u_{1}-\frac{c_{y}}{m} \dot{y} \\
& \ddot{z}=\frac{1}{m}(\cos \phi \cos \theta) u_{1}-g-\frac{c_{z}}{m} \dot{z}, \\
& \ddot{\phi}=\frac{J_{y}-J_{z}}{J_{x}} \dot{\theta} \dot{\psi}-\frac{J_{r}}{J_{x}} \dot{\theta} \Omega_{n}-\frac{b_{\phi}}{J_{x}} \dot{\phi}+\frac{1}{J_{x}} u_{2}, \\
& \ddot{\theta}=\frac{J_{z}-J_{x}}{J_{y}} \dot{\phi} \dot{\psi}+\frac{J_{r}}{J_{y}} \dot{\phi} \Omega_{n}-\frac{b_{\theta}}{J_{y}} \dot{\theta}+\frac{1}{J_{y}} u_{3}, \\
& \ddot{\psi}=\frac{J_{x}-J_{y}}{J_{z}} \dot{\phi} \dot{\theta}-\frac{b_{\psi}}{J_{z}} \dot{\psi}+\frac{1}{J_{z}} u_{4},
\end{aligned}
$$

donde $m$ es la masa del robot, $g$ es la constante de la aceleración de la gravedad y $u_{1}$ es la entrada principal de fuerza que engloba los términos $F_{1}, F_{2}, F_{3}$ y $F_{4}$ y un coeficiente de empuje $b$. En el diagrama esquemático de cuerpo libre mostrado en la Fig. 1, se aprecia que el movimiento en la dirección $x$ se puede regular mediante el desplazamiento angular $\theta$, para la dirección $y$ la regulación es de $\phi$ y el control de la posición en dirección $z$ denominada altitud, se consigue mediante la manipulación de las fuerzas producidas por cada rotor.

Las entradas de control $u_{2}, u_{3}$ y $u_{4}$ se producen debido a la diferencia de fuerzas producidas por cada rotor y la relación de estas con un coeficiente de arrastre $d$, que corresponden de manera directa con los movimientos de roll, pitch y yaw respectivamente y se utilizan como las entradas de par de control del sistema; $\Omega_{n}$ es la suma algebraica de las velocidades angulares y se observa en la ecuación (7), ésta contribuye a los efectos giroscópicos provocados por la rotación de las aspas de los motores, en diversos trabajos se presenta como una perturbación que debe ser compensada por el controlador propuesto; el momento de inercia del rotor se expresa mediante $J_{r}$. Debido a que la estructura del robot se considera simétrica, solamente se presentan los elementos de la diagonal del tensor de inercia $J_{x x}, J_{y y}$ y $J_{z z}$

$$
\Omega_{n}=-\omega_{1}+\omega_{2}-\omega_{3}+\omega_{4}
$$

Los parámetros $b_{r}$ son los coeficientes de amortiguamiento rotacional para $r=\phi, \theta, \psi$, en tanto $c_{n}$ son los coeficientes de amortiguamiento traslacional para $n=x, y, z$, los coeficientes de empuje y arrastre, $b$ y $d$ respectivamente, relacionan idealmente a las entradas principales de fuerza y par con el cuadrado de las velocidades angulares de cada motor $\omega_{1}, \omega_{2}, \omega_{3}$ y $\omega_{4}$, estos coeficientes pueden ser aproximados mediante la teoría del elemento pala. 


\section{Un esquema de control basado en el seguimiento de trayectorias de referencia}

El objetivo del esquema de control presentado en este trabajo, es la regulación y seguimiento de trayectorias de referencia planificadas para los movimientos de rotación y traslación del Quadrotor. El esquema de control propuesto esta estructurado en dos etapas principales como se muestra en la Fig. 2. La primera etapa del esquema de control se basa en el seguimiento de los desplazamientos en las direcciones $X$ y $Y$, donde se determinan los desplazamientos angulares de referencia para $\theta$ y $\phi$. La segunda etapa se encarga del seguimiento de las trayectorias establecidas para $z, \phi, \theta$ y $\psi$.

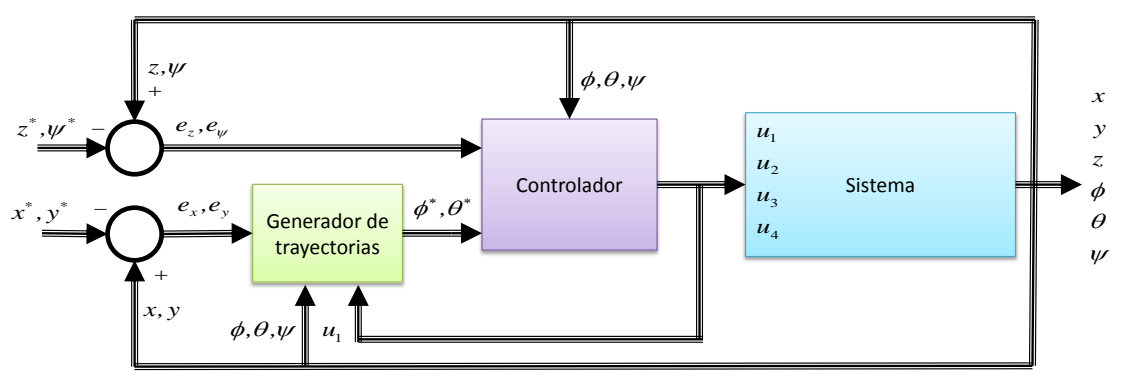

Fig. 2. Diagrama de bloques del esquema de control.

En el esquema de la Fig. 2 se presenta la primera etapa del esquema de control como un bloque generador de trayectorias que determina los desplazamientos angulares de referencia $\theta^{*}$ y $\phi^{*}$, para el correcto seguimiento de las trayectorias planificadas mediante (8) y (9), las expresiones se deducieron de (1) y (2)

$$
\begin{gathered}
\phi^{*}=\sin ^{-1}\left(\frac{m}{u_{1}}\left[\left(v_{x}+\frac{c_{x}}{m} \dot{x}\right) \sin \psi-\left(v_{y}+\frac{c_{y}}{m} \dot{y}\right) \cos \psi\right]\right), \\
\theta^{*}=\sin ^{-1}\left(\frac{m v_{x}+c_{x} \dot{x}}{u_{1} \cos \phi \cos \psi}-\frac{\sin \phi \sin \psi}{\cos \phi \cos \psi}\right),
\end{gathered}
$$

aquí, los controladores auxiliares $v_{x}$ y $v_{y}$, presentan la estructura expresada en la ecuación (10)

$$
v_{k}=-\beta_{2} \dot{e_{k}}-\beta_{1} e_{k}-\beta_{0} \int_{0}^{t} e_{k} d t
$$


para $k=x, y$; donde los errores de seguimiento para cada dirección se define como: $e_{x}=x-x^{*}$ y $e_{y}=y-y^{*}$. Aquí el * indica las trayectorias planificadas para el Quadrotor. Por lo tanto las dinámicas de los errores de seguimiento en lazo cerrado están dadas por

$$
\ddot{e}_{k}^{(3)}+\beta_{2, k} \ddot{e}_{k}+\beta_{1, k} \dot{e}_{k}+\beta_{0, k} e_{k}=0 .
$$

En cuanto a las segunda etapa se tiene que el controlador $u_{1}$ se encarga de la regulación y seguimiento de la trayectoria vertical del sistema en la dirección $Z$ y se relaciona directamente con la suma total de las fuerzas producidas por los rotores. Los controladores propuestos $u_{2}$ y $u_{3}$ están relacionados con los movimientos de roll y pitch, su regulación y seguimiento son de suma importancia ya que de ellos depende el control de desplazamiento en las direcciones $X$ y $Y$, por su parte $u_{4}$ hace referencia al movimiento de yaw, las expresiones para estos controladores son

$$
\begin{aligned}
& u_{1}=\frac{1}{\cos \phi \cos \theta}\left(m v_{z}+c_{z} \dot{z}+m g\right), \\
& u_{2}=J_{x} v_{\phi}-\left(J_{y}-J_{z}\right) \dot{\theta} \dot{\psi}+J_{r} \dot{\theta} \Omega_{n}+b_{\phi} \dot{\phi}, \\
& u_{3}=J_{y} v_{\theta}-\left(J_{z}-J_{x}\right) \dot{\phi} \dot{\psi}-J_{r} \dot{\phi} \Omega_{n}+b_{\theta} \dot{\theta}, \\
& u_{4}=J_{z} v_{\psi}-\left(J_{x}-J_{y}\right) \dot{\phi} \dot{\theta}+b_{\psi} \dot{\psi},
\end{aligned}
$$

donde los controladores $v_{z}, v_{\phi}, v_{\theta}$ y $v_{\psi}$, presentan la estructura en (16) con $p=$ $z, \phi, \theta, \psi$. Los errores de seguimiento están definidos como: $e_{z}=z-z^{*}, e_{\phi}=$ $\phi-\phi^{*}, e_{\theta}=\theta-\theta^{*}$ y $e_{\psi}=\psi-\psi^{*}$. Por lo tanto los controladores se definen como

$$
v_{p}=-\beta_{2, p} \dot{e}_{p}-\beta_{1, p} e_{p}-\beta_{0, p} \int_{0}^{t} e_{p} d t .
$$

Las ganancias de control $\beta_{i, j}, i=0,1,2, j=x, y, z, \phi, \theta, \psi$, se deben de seleccionar para que los polinomios característicos asociados a la expresión (11) sean polinomios Hurwitz (estables).

\section{Resultados}

Para validar el desempeño del enfoque de control propuesto, se realizaron diversas simulaciones mediante el uso de métodos numéricos, donde $g=9.81$ $\mathrm{m} / \mathrm{s}, m=0.973 \mathrm{~kg}, J_{r}=0.0000286 \mathrm{Nms}^{2}, J_{x}=J_{y}=0.00866 \mathrm{Nms}^{2}, J_{z}=$ $0.00866 \mathrm{Nms}^{2}, c_{x, y, z}=0.0001 \mathrm{Ns} / \mathrm{m}$ y $b_{\phi, \theta, \psi}=0.0001 \mathrm{Nms}$. En la Fig. 3(a) se observan las trayectorias planificadas y el seguimiento realizado por el Quadrotor en cada una de las direcciones en el plano cartesiano, la trayectoria planificada está definida para la dirección $Y$ como

$$
y^{*}=r_{y} \sin \left(\omega_{y} t\right)
$$

para la dirección $X$

$$
x^{*}=r_{x} \sin \left(\omega_{x} t\right),
$$


donde los radios $r_{x, y}=0.5 \mathrm{~m}$ y las velocidades angulares $\omega_{x, y}=0.1 \mathrm{rad} / \mathrm{s}$. En cuanto a $Z$, la trayectoria deseada es

$$
z^{*}=r_{z} \sin \left(\omega_{z} t\right)
$$

con un radio $r_{z}=0.5 \mathrm{~m} \mathrm{y} \omega_{z}=0.3 \mathrm{rad} / \mathrm{s}$. La referencia $\psi^{*}=0.5 \mathrm{rad}$ para $t>0 \mathrm{~s}$. Los valores de las ganancia se definieron como: $\beta_{0, i}=18.5, \beta_{1, i}=$ $110, \beta_{2, i}=250$, para $i=x, y, z$ y $\beta_{0, j}=45000, \beta_{1, j}=3450, \beta_{2, j}=101$, para $j=\phi, \theta, \psi$. En la Fig. 3(b) se observa que el vehículo aéreo con cuatro rotores tiene la capacidad de hacer el seguimiento de manera adecuada, el algoritmo de control le permite seguir la referencia de las trayectorias planificadas de manera suave y debido a las características del diseño del controlador el error converge a cero. Un aspecto importante a considerarse en el análisis mediante simulación, son las magnitudes de las señales de control ya que existen limitaciones físicas por parte de los actuadores en la entrega de fuerzas y pares al sistema.

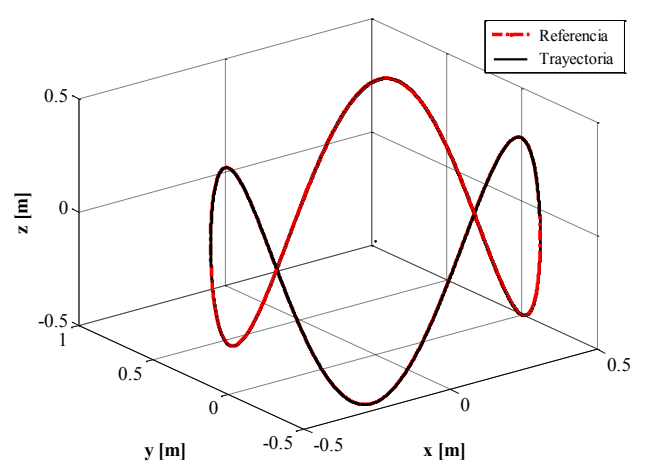

(a)
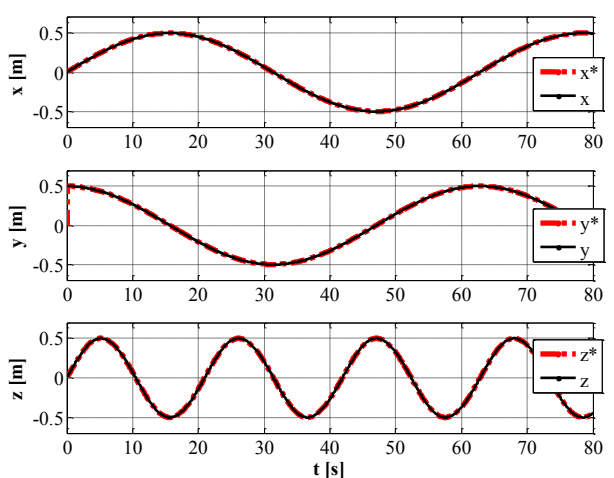

(b)

Fig. 3. Seguimiento de trayectoria en las direcciones $X, Y$ y $Z$. 
En la Fig. 4 se observa que las respuestas de los controladores principales, no presentan respuestas abruptas que pudieran verse limitadas en la implementación, con propósitos de presentación se analizan durante $2 \mathrm{~s}$. La señal de control $u_{4}$ permanece en cero después de alcanzar la referencia establecida $\psi=0.5 \mathrm{rad}$. Por su parte $u_{1}$ se observa que siempre esta actuando contra la fuerza de gravedad, evitando que el helicóptero entre en contacto con el suelo. El seguimiento de las trayectorias planificadas para los ángulos de referencia, establecidos por el generador de trayectorias se aprecia en la Fig. 5 y la Fig. 6, se observa el comportamiento del sistema bajo la acción de los controladores; es claro apreciar que el controlador tiene la capacidad de que el sistema alcance las referencias en tiempo finito. Sin embargo, se debe de ser cautelosos cuando se determinan las ganancias del controlador ya que las señales de control podrían saturar el sistema, lo que no es deseable en la implementación de laboratorio.

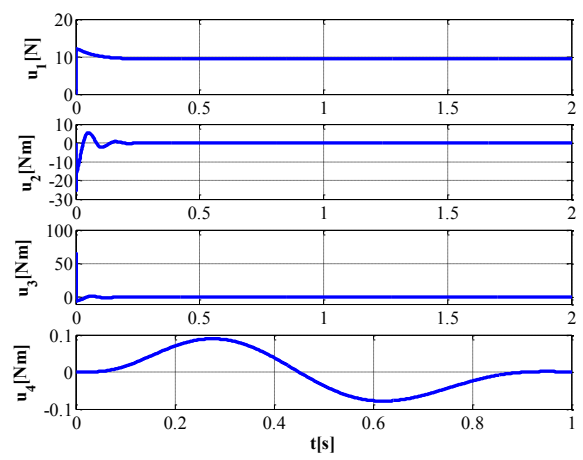

Fig. 4. Señales de control.

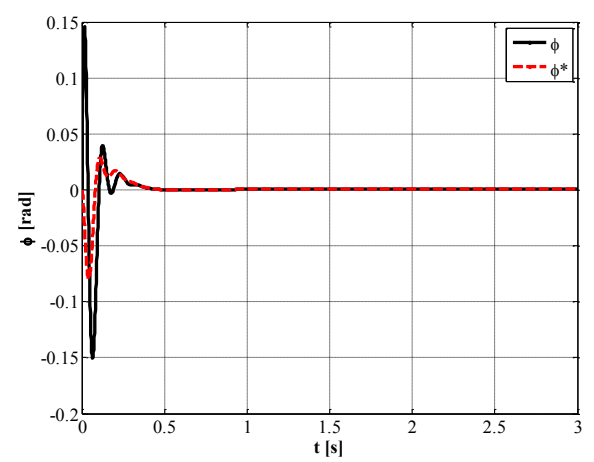

Fig. 5. Seguimiento de la trayectoria planificada de $\phi$. 


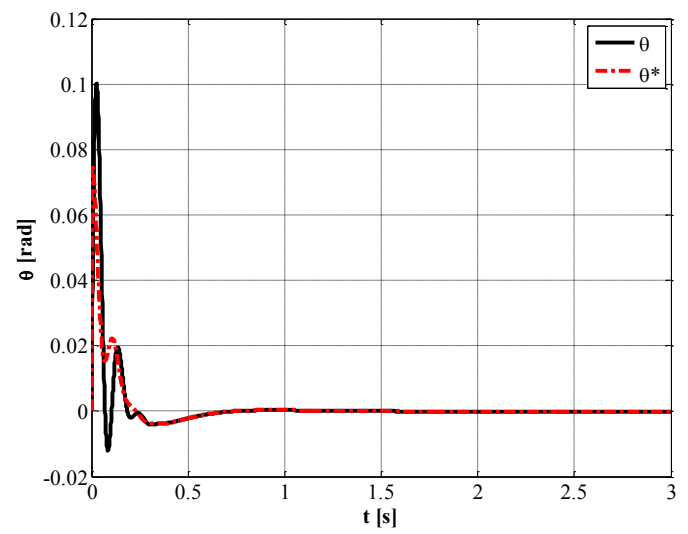

Fig. 6. Seguimiento de la trayectoria planificada de $\theta$.

\section{Conclusiones}

En este artículo se ha descrito un esquema de control para tareas de regulación y seguimiento de trayectorias planificadas para el movimiento de un helicóptero con cuatro rotores. El enfoque de control propuesto es capaz de seguir trayectorias variables planificadas de manera eficiente. Además, la implementación del esquema de control propuesto es relativamente simple, obteniendo un desempeño aceptable para la regulación y seguimiento de trayectorias planificadas para todas las variables de estado que describen la configuración de movimiento de un Quadrotor. En futuros trabajos se consideraran la influencia de posibles perturbaciones, afectando la dinámica del sistema, en el diseño y evaluación de esquemas de control de movimiento.

\section{Referencias}

1. Corke, P.I.: Robotics, vision and control: fundamental algorithms in MATLAB. Springer, Berlin (2011)

2. Alderete, T.S.: Simulator Aero Model Implementation. Technical Paper, pp. 1-21 (1997)

3. Castillo, P., García, P., Lozano, R., Albertos, P.: Modelado y estabilización de un helicóptero con cuatro rotores. Revista Iberoamericana de Automática E Informática Industrial RIAI, 4(1), pp. 41-57 (2007)

4. Bouabdallah, S., Murrieri, P., Siegwart, R.: Design and control of an indoor micro quadrotor. In: Proceedings of the IEEE International Conference on Robotics and Automation ICRA '04, Vol. 5, pp. 4393-4398 (2004)

5. Bouabdallah, S., Noth, A., Siegwart, R.: PID vs LQ control techniques applied to an indoor micro quadrotor. In: Proceedings of the IEEE/RSJ International Conference In Intelligent Robots and Systems, (IROS 2004), Vol. 3, pp. 2451-2456 (2004) 
6. Fernando, H.C.T.E., Silva, A.T.A.De, Zoysa, M.D.C.De, Dilshan, K.A.D.C., Munasinghe, S.R.: Modelling, simulation and implementation of a quadrotor UAV. In: Proceedings of the IEEE 8th International Conference on Industrial and Information Systems ICIIS , pp. 207-212 (2013)

7. Johnson, N.L., Leang, K.K.: Enhanced Proportional-Derivative Control of a Micro Quadcopter. In: Proceedings of the ASME Dynamic Systems and Controls Conference, pp. 1-5 (2013)

8. Erginer, B., Altug, E.: Modelling and PD Control of a Quadrotor VTOL Vehicle. In: Proceedings of the IEEE Intelligent Vehicles Symposium, pp. 894-899 (2007)

9. Leong, B.T.M., Low, S.M., Ooi, M.P.L.: Low-cost microcontroller-based hover control design of a quadcopter. In: Proceedings of the International Symposium on Robotics and Intelligent Sensors (IRIS), Vol. 41, pp. 458-464 (2012)

10. Bouabdallah, S., Siegwart, R.: Backstepping and sliding-mode techniques applied to an indoor micro Quadrotor. In: Proceedings of the IEEE International Conference on Robotics and Automation, pp. 224-2252 (2005)

11. Ha, C., Zuo, Z., Choi, F. B., Lee, D.: Passivity-based adaptive backstepping control of quadrotor-type UAVs. Robotics and Autonomous Systems, 62(9), pp. $1305-1315$ (2014)

12. Zheng, E.H., Xiong, J.J., Luo, J.L.: Second order sliding mode control for a quadrotor UAV. ISA Transactions, 53(4), pp. 1-7 (2014)

13. Luque-Vega, L., Castillo-Toledo, B., Loukianov, A. G.: Robust block second order sliding mode control for a quadrotor. Journal of the Franklin Institute, 349(2), pp. $719-739$ (2012)

14. Raffo, G.V., Ortega, M.G., Rubio, F.R.: An integral predictive/nonlinear $H \infty$ control structure for a quadrotor helicopter. Automatica, 46(1), pp. 29-39 (2010)

15. Raharja, N.M., Iswanto, Faris, M., Cahyadi, A.I.: Hover position quadrotor control with fuzzy logic. In: Proceedings of the 0st International Conference on Information Technology, Computer and Electrical Engineering (ICITACEE), pp. 89-92 (2014)

16. Emran, B.J., Yesildirek, A.: Robust Nonlinear Composite Adaptive Control of Quadrotor. International Journal of Digital Information and Wireless Communications, 4(2), pp. 213-225 (2014)

17. Hsu, C.F., Chang, C.W.: Intelligent dynamic sliding-mode neural control using recurrent perturbation fuzzy neural networks. Neurocomputing, 173, pp. 734-743 (2016)

18. Liu, H., Li, D., Zuo, Z., Zhong, Y.: Robust three-loop trajectory tracking control for quadrotors with multiple uncertainties. IEEE Transactions on Industrial Electronics, 63(4), pp. 2263-2274 (2016)

19. Sira-Ramírez, H.: On the linear control of the quad-rotor system. In: Proceedings of the 2011 American Control Conference. pp. 3178-3183 (2011)

20. Aguilar-Ibáñez, C., Sira-Ramírez, H., Suárez-Castañón, M.S., Martínez-Navarro, E., Moreno-Armendariz, M.A.: The trajectory tracking problem for an unmanned four-rotor system: flatness-based approach. Int. J. Control. 85, pp. 69-77 (2012)

21. Mian, A.A., Mian, I.A., Wang, D.: Backstepping based PID control strategy for an underactuated aerial robot. In: IFAC Proceedings (IFAC-PapersOnline), Vol. 17, pp. 15636-15641 (2008)

22. Fatan, M., Sefidgari, B.L., Barenji, A.V.: An adaptive neuro PID for controlling the altitude of quadcopter robot. In: Proceedings of the IEEE 18th International Conference on Methods \& Models in Automation \& Robotics (MMAR), pp. 662-665 (2013) 